\title{
In vitro characterization of a nitro-forming oxygenase involved in 3- (trans-2'-aminocyclopropyl)alanine biosynthesis
}

\author{
Linlin Pang†, Weijing Niu†, Yuwei Duan†, Liujie Huo ${ }^{\dagger}$, Aiying $\mathrm{Li}^{\dagger}$, Jiequn $\mathrm{Wu}^{\S}$, Youming Zhang†, \\ Xiaoying Bian ${ }^{t, *}$, and Guannan Zhongt,‡,*
}

$\dagger$ Helmholtz International Lab for AntiInfectives, Shandong University-Helmholtz Institute of Biotechnology, State Key Laboratory of Microbial Technology, Shandong University, Qingdao 266237, China.

‡ Suzhou Institute of Shandong University, Suzhou 215123, China.

$\S$ Collaborative Innovation Center of Yangtze River Delta Region Green Pharmaceuticals, College of Pharmaceutical Sciences, Zhejiang University of Technology, Hangzhou 310014, China.

Supporting Information Placeholder

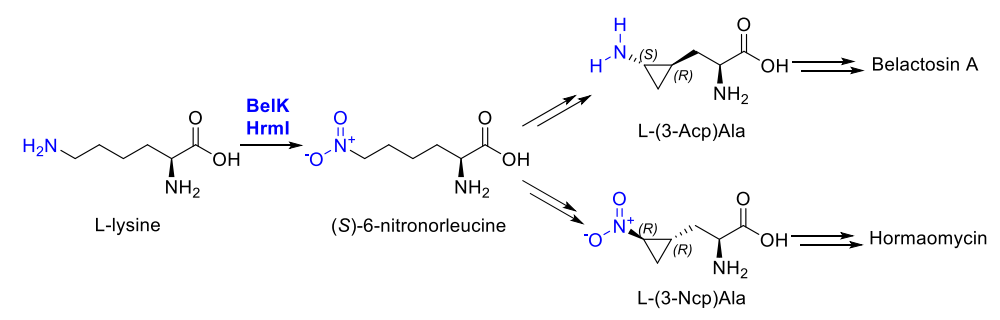

\begin{abstract}
In vitro characterization experiments revealed the formations of 3-(trans-2'-aminocyclopropyl)alanine ((3Acp)Ala) and 3-(trans-2'-nitrocyclopropyl)alanine ((3-Ncp)Ala) are originated via two homologous proteins BelK and HrmI, which regioselectively catalyze $\mathrm{N} \varepsilon$-nitration of L-lysine. The two enzymes belong to the emerging heme-oxygenase-like diiron oxidase and oxygenase (HDO) superfamily and the catalytic center of BelK is validated by homology modeling and site-directed mutations. Based on the in vitro characterization, the biosynthetic pathways of (3-Acp)Ala and (3-Ncp)Ala are first proposed.
\end{abstract}

Cyclopropane moiety has been found in a number of natural products of secondary metabolism from plants, fungi, and microorganisms. ${ }^{1}$ The cyclopropyl skeleton lacks the ability to freely rotate and serves as a rigid structural element, together with the $\mathrm{C}-\mathrm{C}-\mathrm{C}$ bond angle much different than the ideal bond angle of $109.5^{\circ}$, resulting in a ring strain up to $27.6 \mathrm{kcal} / \mathrm{mol}$ of cyclopropane. ${ }^{2}$ The cyclopropyl ring is a versatile player that frequently appears in preclinical/clinical drug molecules since it could enhance potency, reduce off-target effects, increase metabolic stability and brain permeability, decrease plasma clearance, alter drug pKa to reduce its P-glycoprotein efflux ratio, etc. ${ }^{3}$ Owing to the unique structure and the excellent contributions to drug molecules, cyclopropyl ring has led to advances in elegant synthetic methods. ${ }^{4}$

Belactosin A (1, Figure 1), a natural product isolated from Streptomyces sp. UCK 14 in 2000, has been shown to bind covalently to the proteasome $\beta_{5}$ subunit and prevent cell cycle division in tumor cells at the G2/M stage, representing a novel lead for developing potent anticancer agents. ${ }^{5,6}$ The most intriguing structural feature of belactosin $\mathrm{A}$ is the 3(trans-2'-aminocyclopropyl)alanine ((3-Acp)Ala) (3) moiety. A similar chemical structure named 3-(trans-2'- nitrocyclopropyl)alanine ((3-Ncp)Ala) (4) with the opposite cyclopropyl stereoconfigurations is found in the cyclooctapeptide hormaomycin (2) (also known as takaokamycin, Figure 1) produced by Streptomyces griseoflavus W384 that functions as a bacterial signaling metabolite and a narrow spectrum antibiotic. ${ }^{7,8}$ Isotope labeling studies established that (3-Ncp)Ala was derived from L-lysine (5), yet the exact biosynthetic mechanism for cyclopropanation still remained elusive. Herein, we address that the iron-containing redox enzymes BelK and HrmI act as novel N-oxygenases, regioselectively convert the $\varepsilon$-amino of L-lysine to nitro group and initiate the biosyntheses of cyclopropyl rings.

Our comparative analysis of the available biosynthetic gene clusters of belactosin $\mathrm{A}(\mathrm{bel})^{10}$ and hormaomycin $(\mathrm{hrm})^{11}$ (Figure S1) revealed three highly conserved genes, i.e., belK and hrmI (encoding iron-containing redox enzymes), belL and hrmJ (encoding non-heme iron and $\alpha$-ketoglutaric acid ( $\alpha-\mathrm{KG})$ dependent oxygenases), belM and hrmT (encoding diaminopimelate epimerases) (Table S1). Given the structural similarity between (3-Acp)Ala and (3Ncp)Ala, these genes mentioned above were deduced to take part in the biosyntheses of cyclopropyl rings. 
Not only that, the peptide backbone in hormaomycin is assembled by nonribosomal peptide synthetases (NRPSs), whereas in belactosin $\mathrm{A}$ is assembled by discrete amide ligases. ${ }^{10,11}$ Taken into account the wide differences in the biosyntheses and chemical structures between belactosin A and hormaomycin, it is very likely that (3-Acp)Ala and (3Ncp)Ala are biosynthesized separately from other parts. Diaminopimelate epimerases catalyze the penultimate step during L-lysine biosynthesis in most plants, bacteria, and lower fungi. ${ }^{12}$ Despite the lack of experimental evidence, BelM and HrmT have been speculated to play a role in diverting lysine precursors from primary metabolism to secondary metabolism. ${ }^{11}$ Therefore, BelK/HrmI and BelL/HrmJ are the most likely candidates for the cyclopropane moieties biosyntheses.
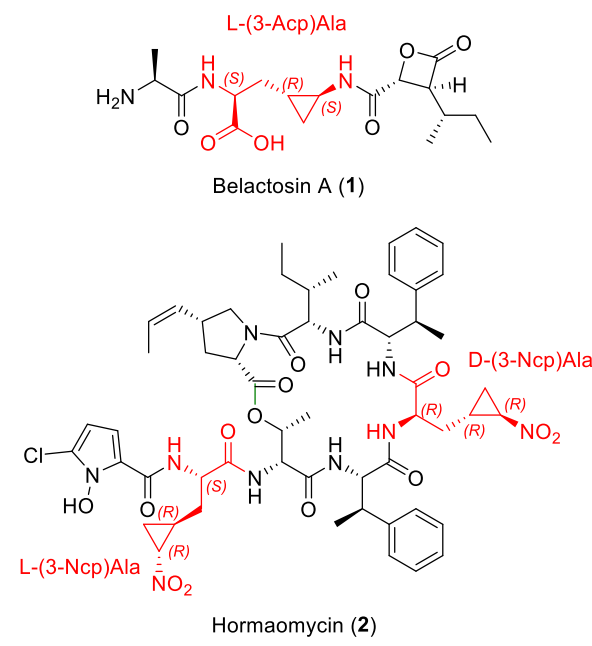

Figure 1. Chemical structures of belactosin A (1) and hormaomycin (2). (3-Acp)Ala and (3-Ncp)Ala are highlighted in red.

Next, we heterologously expressed and purified the nonheme iron and $\alpha$-KG dependent oxygenase BelL (Figure S2). However, in vitro characterization of BelL in the presence of L-lysine, $\alpha$-KG, $\mathrm{FeSO}_{4} \cdot 7 \mathrm{H}_{2} \mathrm{O}$, L-ascorbic acid and dithiothreitol (DTT) showed no potential product under different conditions, neither for the homologous protein HrmJ. This excluded the possibility that BelL or HrmJ was the first enzyme converting L-lysine to cyclopropyl rings and let us focus on the other oxygenases BelK and HrmI.

The structure of BelK was predicted online using the ITASSER approach, ${ }^{13-15}$ which revealed high similarity to the crystal structure of SznF (PDB: 6M9R) from streptozotocin biosynthetic gene cluster in spite of a very low sequence identity (14.8\%) (Figure S3). SznF is an iron-containing redox enzyme as well and participates in $N$-nitrosourea formation. X-ray structure showed that SznF contained an Nterminal dimerization domain, a central helical bundle domain and a C-terminal cupin domain. ${ }^{16,17}$ The latter domain is lack in the BelK predicted structure. In vitro characterization of BelK was performed according to SznF using reduced nicotinamide adenine dinucleotide (NADH) and phenazine methosulfate (PMS) as the reductant and electron transfer (Figure S4). After dansyl chloride (DNSC) derivatization and high performance liquid chromatography electrospray ionization high resolution mass spectrometry (HPLC-ESIHRMS) analysis, a new peak (8) with a longer retention time and 30 Da mass increase $\left([\mathrm{M}+\mathrm{H}]^{+} \mathrm{m} / \mathrm{z}\right.$ : calcd. 410.1380 for $\left[\mathrm{C}_{18} \mathrm{H}_{24} \mathrm{~N}_{3} \mathrm{O}_{6} \mathrm{~S}\right]^{+}$, found 410.1385$)$ was detected in addition to DNS-Lys (7, [M+H] ${ }^{+} m / z$ : calcd. 380.1639 for $\left[\mathrm{C}_{18} \mathrm{H}_{26} \mathrm{~N}_{3} \mathrm{O}_{4} \mathrm{~S}\right]^{+}$, found 380.1642) (Figure 2B and S5). This compound disappeared if any of the L-lysine, NADH, PMS or BelK was omitted, indicating that all the elements above were necessary for the assay. Interestingly, a same result was found when HrmI was used instead of BelK, implying that the differences between (3-Acp)Ala and (3-Ncp)Ala are not determined by BelK or HrmI.

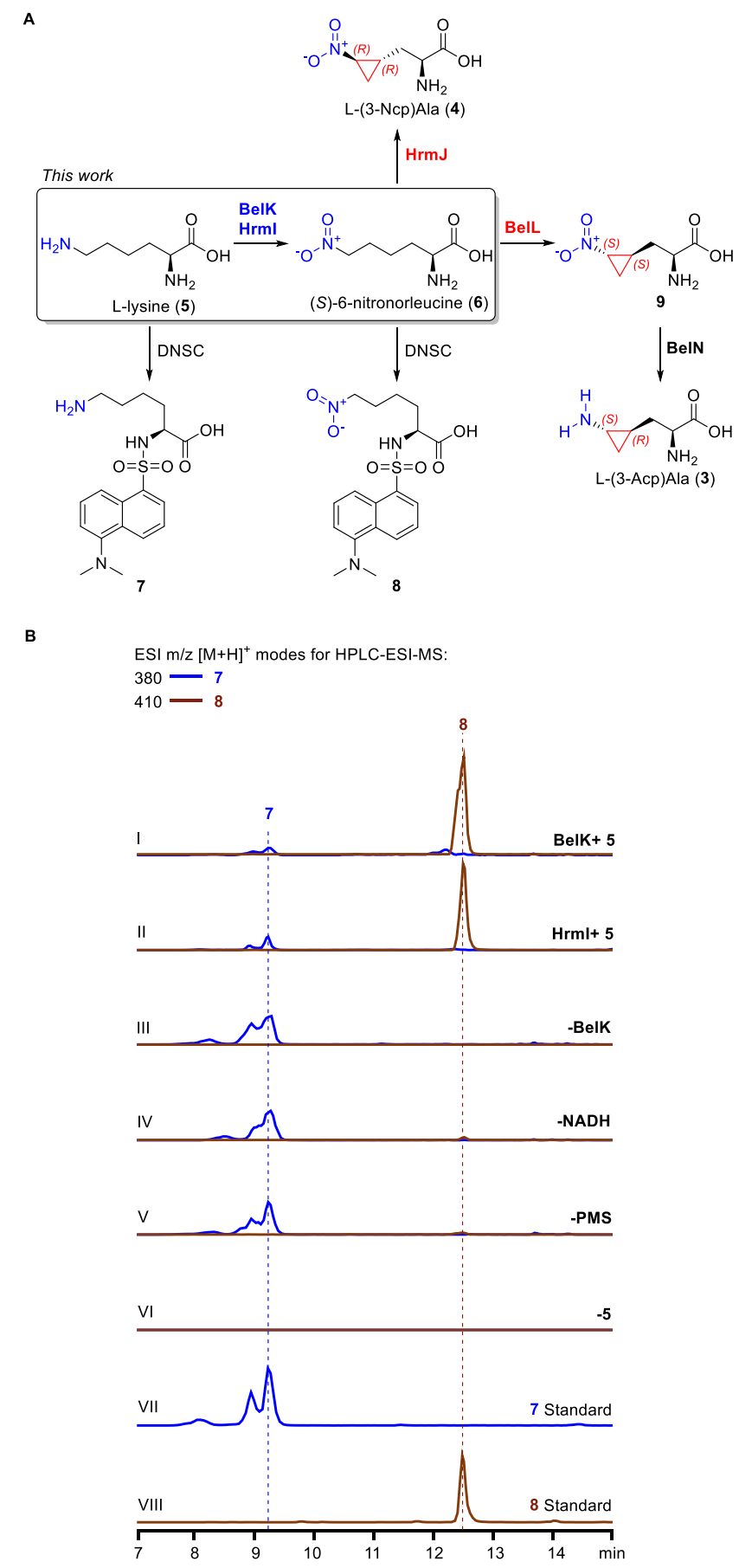

Figure 2. In vitro characterization of BelK and HrmI. (A) Proposed biosynthetic pathways of (3-Acp)Ala and (3Ncp)Ala. (B) Validation of the $\mathrm{N} \varepsilon$-oxygenase activity of BelK and HrmI. 
It is worth mentioning that $\mathbf{8}$ had two more oxygens, two less hydrogens but exhibited less polarity than 7 . Thus, we hypothesized that BelK/HrmI converted $\varepsilon$-amino in L-lysine to nitro group, since the $\alpha$-amino of L-lysine was preserved in both (3-Acp)Ala and (3-Ncp)Ala. To validate this hypothesis, $(S)$-6-nitronorleucine (6) was synthesized according to a previously reported 4 -step procedure (Figure S6). ${ }^{18}$ DNSC derivatized 6 displayed the identical mass and retention time with 8 (Figure 2B), confirming BelK and HrmI are $\mathrm{N} \varepsilon$ oxygenases and oxygenate the nitration of the L-lysine $\varepsilon$ amino group (Figure 2A). L-ornithine (10) was employed as the L-lysine analog to evaluate the substrate tolerance of BelK. However, no product was detected after DNSC derivatization (Figure S7), indicating that the length of carbon chain might be crucial for BelK recognition.

Distinct from the ferritin-like diiron oxidase and oxygenase (FDO) superfamily such as AurF, CmlI, ObiL and EtmA, Unk1D, Unk2D that belong to the Pfam 11583, ${ }^{19-23}$ BelK and HrmI are placed in the Pfam 14518 as the non-heme diiron oxygenase SznF. ${ }^{24,25}$ Phylogenetically, BelK and HrmI fall into a clade close to SznF but far away from FDO superfamily (Figure 3). Apart from SznF, other enzymes such as UndA (PDB: 4WWZ) involved in the biosynthesis of 1-undecene in Pseudomonas aeruginosa and Chlamydia protein associating with death domains (CADD) (PDB: 1RCW) implicated in the biosynthesis of para-aminobenzoic acid in Chlamydia trachomatis also constructed a similar helical bundle topology like SznF. ${ }^{26-30}$ These proteins together with the diiron N-oxygenase RohS involved in azomycin biosynthesis and the olefin-forming desaturase BesC taken part in L- $\beta$ ethynylserine biogenesis constituted the emerging hemeoxygenase-like diiron oxidase and oxygenase (HDO) superfamily. ${ }^{25,31,32}$ As new members of the HDO superfamily, it's noteworthy that BelK and HrmI are the only ones in this new superfamily which containing an $\mathrm{N}$-terminal dimerization domain besides SznF. Attempt to remove the N-terminal domain of BelK resulted in no expression, pointing out that the $\mathrm{N}$-terminal domain is important for BelK expression. Moreover, BelK and HrmI are quite different from other diiron nitro-forming enzymes uncovered to date. Since most of the diiron nitro-forming enzymes act on aryl amines (AurF, CmlI, ObiL and RohS) or carrier-bound amino acids (EtmA and its homologues) (Figure S8 and S9). To the best of our knowledge, BelK and HrmI are the first diiron nitro-forming enzymes that act on the free amino acids.

Homology modeling of BelK performed by I-TASSER identified the presumed diiron binding residues E205, H215, E269, H299, D303 and H306 that are highly conserved in all BelK-like proteins (Figure S3), e.g., HrmI, SagK from S. agglomeratus 6-3-2 (sag cluster), SctK from $S$. chrestomyceticus TBRC 1925 (sct cluster), McnK from Micromonospora sp. CNZ322 (mcn cluster) and NcsK from Nocardia sp. CS682 (ncs cluster) (Figure S1 and S10). Mutagenesis of any of the six predicted ligands completely abolished the activity of BelK, validating the necessity of the presumed diiron binding residues (Figure 4). In addition to the six ligands, a tyrosine residue (Y295 in BelK) near the active sites is also conserved in all BelK-like proteins, SznF and CADD (Figure S3, S10 and S11). It's surprising a single mutation of Y295 to alanine resulted in the insolubility of BelK, indicating that Y295 plays a particularly prominent role in the proper architecture of BelK. To further verify the function of this tyrosine residue, $\mathrm{Y} 295 \mathrm{~F}$ was constructed. In contrast to Y295A, Y295F was quite soluble nevertheless lost the catalytic activity (Figure 4 and S4), implying that the hydroxyl group in Y295 might be vital for the correct function of BelK. Due to the instability of the diiron catalytic center of many HDO proteins, an extra coordinating side chain of E189 in SznF was identified very recently, ${ }^{25}$ whereas the corresponding residue at this position is phenylalanine (F179 for BelK) in all BelK-like proteins just like UndA and CADD (Figure S3, S10 and S11). It's quite difficult to identify the extra coordinating residue in BelK for the present. It isn't remotely possible that the carboxylate group of L-lysine may act as the extra coordination in all the BelK-like proteins, just like UndA. ${ }^{25}$

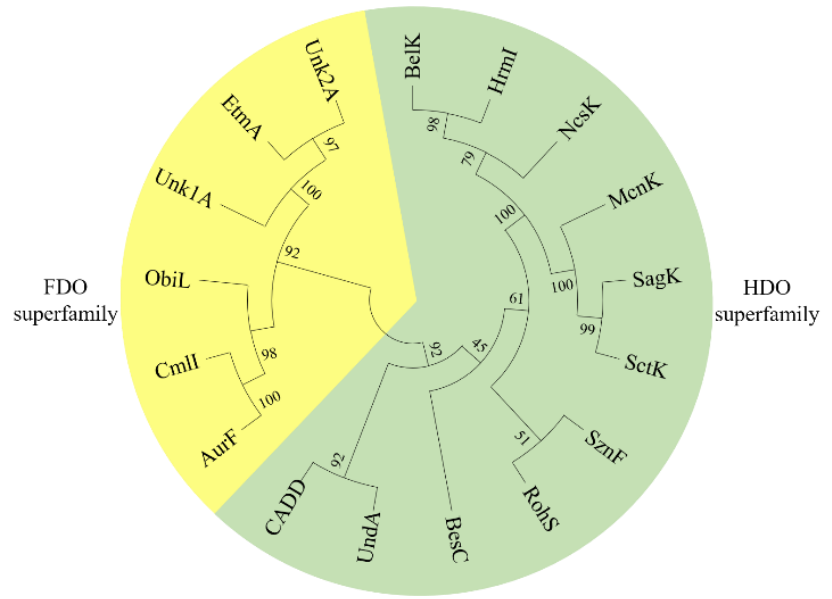

Figure 3. Phylogenetic analysis of the FDO and HDO superfamily proteins. The tree was constructed with the neighbor-joining method and a bootstrap test with 2000 iterations.

The following part of (3-Acp)Ala and (3-Ncp)Ala biosyntheses remains to be elucidated. It's likely that the nonheme iron and $\alpha-K G$ dependent oxygenases BelL and HrmJ are responsible for the formations of cyclopropyl rings with opposite stereoconfigurations (Figure 2A). The NE-nitro group is anticipated to be essential for cyclopropanation, for no products were detected in the BelL or HrmJ assays with L-lysine as the substrate. While this manuscript was under preparation, a non-heme iron and $\alpha$-KG dependent oxygenase TqaL was reported to catalyze the aziridine biogenesis from L-valine and expand the catalytic repertoire of the Fe(II) $/ \alpha-$ KG oxygenases (Figure S12)..$^{33}$ Considering that BelL, HrmJ and TqaL are placed in the same Pfam (Pfam 10014), the result of TqaL supports the hypothesis that BelL and HrmJ catalyze the generation of cyclopropyl rings.

It appears that a nitroreductase absent in $\mathrm{hrm}$ cluster is required for the nitro reduction of 9 to obtain (3-Acp)Ala (Figure 2A). In silico analysis unveiled the genes belN and its homologues (encoding molybdopterin oxidoreductases), which in fact did exist in sag, sct, mcn and ncs biosynthetic gene clusters (Figure S1). Besides belK/L/M/N, no other homologous genes were found in bel and the four clusters mentioned above, surmising that the four genes are sufficient to generate (3-Acp)Ala and the products from sag, sct, $m c n$ and $n c s$ clusters might also comprise the (3-Acp)Ala moiety. Further studies are still underway in our laboratory. 


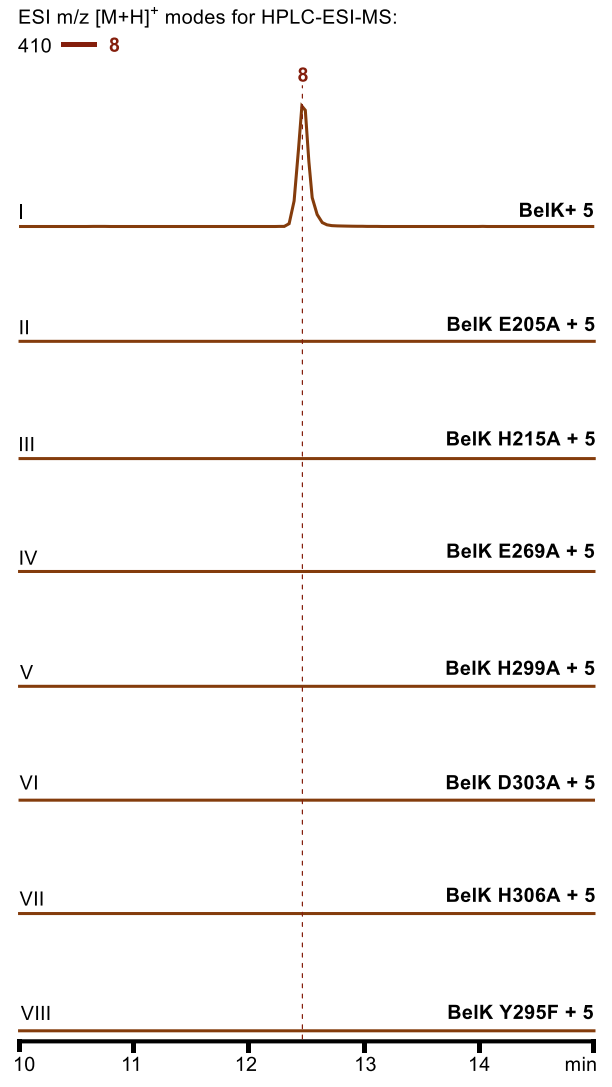

Figure 4. Evaluation of the putative catalytic sites of BelK.

In conclusion, BelK and HrmI, new members of the emerging HDO superfamily, have been biochemically characterized to regioselectively catalyze the unique $\mathrm{N} \varepsilon$-nitration of L-lysine under reducing conditions. It was reported that 6 was an inhibitor for human arginase I and resulted in higher cellular concentrations of L-arginine for NO biosynthesis. ${ }^{34}$ Therefore, this compound could act as a candidate for the treatment of diseases in which upregulated arginase activity compromises NO-dependent physiological processes. Our result provides a new method for the synthesis of 6 in a mild reaction condition. Furthermore, this study highlights the versatile functions of the HDO superfamily, expands the existing knowledge for enzyme-mediated nitro formation, and particularly sheds light on the research of (3-Acp)Ala and (3-Ncp)Ala biogenesis. BelK and its homologues could serve as new genome mining probes for the discovery of novel bioactive natural products containing nitro group or cyclopropyl skeleton.

\section{ASSOCIATED CONTENT}

\section{Supporting Information}

The Supporting Information is available free of charge on the website.

Full experimental details, in silico analysis, SDS-PAGE, homology modeling, MS data, NMR spectra and sequence alignments. (PDF)

\section{AUTHOR INFORMATION}

\section{Corresponding Author}

* G.Z. Email: zhonggn@sdu.edu.cn

*X.B. Email: bianxiaoying@sdu.edu.cn

Notes

The authors declare no competing financial interest.

\section{ACKNOWLEDGMENT}

We thank Drs. Jingyao Qu, Zhifeng Li, Jing Zhu and Haiyan Sui (Core Facilities for Life and Environmental Sciences, State Key laboratory of Microbial Technology, Shandong University) for the detection and characterization by HPLC-ESI-HRMS and NMR, and Prof. Qingfei Zheng (Department of Radiation Oncology and Center for Cancer Metabolism, The Ohio State University) for proofreading the manuscript and helpful discussions. This work was supported by the National Key R\&D Program of China (2019YFA0905700), the National Natural Science Foundation of China (21907057, 32070060), the Shandong Provincial Natural Science Foundation, China (ZR2019JQ11, ZR2019ZD18), the Natural Science Foundation of Jiangsu Province, China (BK20190201), the 111 project (B16030), the Youth Interdisciplinary Innovative Research Group (2020QNQT009), the Future Plan for Young Scholars, and the Fundamental Research Funds (2019GN032) of Shandong University.

\section{REFERENCES}

(1) Wessjohann, L. A.; Brandt, W.; Thiemann, T. Chem. Rev. 2003, $103,1625$.

(2) Stability of cycloalkanes-ring strain. (2021, June 22). Retrieved June 30, 2021, from https://chem.libretexts.org/@go/page/31410.

(3) Talele, T. T. J. Med. Chem. 2016, 59, 8712.

(4) Chen, D. Y. K.; Pouwer, R. H.; Richard, J.-A. Chem. Soc. Rev. 2012, 41, 4631.

(5) Asai, A.; Hasegawa, A.; Ochiai, K.; Yamashita, Y.; Mizukami, T. J. Antibiot. 2000, 53, 81.

(6) Asai, A.; Tsujita, T.; Sharma, S. V.; Yamashita, Y.; Akinaga, S.; Funakoshi, M.; Kobayashi, H.; Mizukami, T. Biochem. Pharmacol. 2004, 67, 227.

(7) Andres, N.; Wolf, H.; Zähner, H.; Rössner, E.; Zeeck, A.; König, W. A.; Sinnwell, V. Helv. Chim. Acta 1989, 72, 426.

(8) Omura, S., Mamada, H., Wang, N.J., Imamura, N., Oiwa, R., Iwai, Y., and Muto, N. J. Antibiot. 1984, 37, 700.

(9) Brandl, M.; Kozhushkov, Sergei I.; Zlatopolskiy, Boris D.; Alvermann, P.; Geers, B.; Zeeck, A.; de Meijere, A. Eur. J. Org. Chem. 2005, 2005, 123.

(10) Wolf, F.; Bauer, J. S.; Bendel, T. M.; Kulik, A.; Kalinowski, J.; Gross, H.; Kaysser, L. Angew. Chem. Int. Ed. 2017, 56, 6665.

(11) Höfer, I.; Crüsemann, M.; Radzom, M.; Geers, B.; Flachshaar, D.; Cai, X.; Zeeck, A.; Piel, J. Chem. Biol. 2011, 18, 381.

(12) Xu, H.; Andi, B.; Qian, J.; West, A. H.; Cook, P. F. Cell Biochem. Biophys. 2006, 46, 43.

(13) Roy, A.; Kucukural, A.; Zhang, Y. Nat. Protoc. 2010, 5, 725

(14) Yang, J.; Yan, R.; Roy, A.; Xu, D.; Poisson, J.; Zhang, Y. Nat. Methods, 2015, 12, 7.

(15) Yang, J.; Zhang, Y. Nucleic Acids Res. 2015, 43, W174.

(16) Ng, T. L.; Rohac, R.; Mitchell, A. J.; Boal, A. K.; Balskus, E. P. Nature 2019, 566, 94.

(17) He, H.-Y.; Henderson, A. C.; Du, Y.-L.; Ryan, K. S. J. Am. Chem. Soc. 2019, 141, 4026.

(18) Zlatopolskiy, B. D.; Radzom, M.; Zeeck, A.; de Meijere, A. Eur. J. Org. Chem. 2006, 2006, 1525.

(19) Choi, Y. S.; Zhang, H.; Brunzelle, J. S.; Nair, S. K.; Zhao, H. Proc. Natl. Acad. Sci. USA 2008, 105, 6858.

(20) Komor, A. J.; Rivard, B. S.; Fan, R.; Guo, Y.; Que, L.; Lipscomb, J. D. J. Am. Chem. Soc. 2016, 138, 7411. 
(21) Schaffer, J. E.; Reck, M. R.; Prasad, N. K.; Wencewicz, T. A. Nat. Chem. Biol. 2017, 13, 737.

(22) Scott, T. A.; Heine, D.; Qin, Z.; Wilkinson, B. Nat. Commun. 2017, 8, 15935.

(23) He, H.-Y.; Ryan, K. S. Nat. Chem. 2021, 13, 599.

(24) McBride, M. J.; Sil, D.; Ng, T. L.; Crooke, A. M.; Kenney, G. E.; Tysoe, C. R.; Zhang, B.; Balskus, E. P.; Boal, A. K.; Krebs, C.; Bollinger, J. M. J. Am. Chem. Soc. 2020, 142, 11818.

(25) McBride, M. J.; Pope, S. R.; Hu, K.; Okafor, C. D.; Balskus, E. P.; Bollinger, J. M.; Boal, A. K. Proc. Natl. Acad. Sci. USA 2021, 118, e2015931118.

(26) Rui, Z.; Li, X.; Zhu, X.; Liu, J.; Domigan, B.; Barr, I.; Cate, J. H. D.; Zhang, W. Proc. Natl Acad. Sci. USA 2014, 111, 18237.

(27) Manley, O. M.; Fan, R.; Guo, Y.; Makris, T. M. J. Am. Chem. Soc. 2019, $141,8684$.

(28) Zhang, B.; Rajakovich, L. J.; Van Cura, D.; Blaesi, E. J.; Mitchell, A. J.; Tysoe, C. R.; Zhu, X.; Streit, B. R.; Rui, Z.; Zhang, W.; Boal, A. K.; Krebs, C.; Bollinger, J. M. J. Am. Chem. Soc. 2019, 141, 14510.

(29) Schwarzenbacher, R.; Stenner-Liewen, F.; Liewen, H.; Robinson, H.; Yuan, H.; Bossy-Wetzel, E.; Reed, J. C.; Liddington, R. C. J. Biol. Chem. 2004, 279, 29320.

(30) Adams, N. E.; Thiaville, J. J.; Proestos, J.; Juárez-Vázquez, A. L.; McCoy, A. J.; Barona-Gómez, F.; Iwata-Reuyl, D.; de Crécy-Lagard, V.; Maurelli, A. T. mBio 2014, 5, e01378-14.

(31) Hedges, J. B.; Ryan, K. S. Angew. Chem. Int. Ed. 2019, 58, 11647.

(32) Marchand, J. A.; Neugebauer, M. E.; Ing, M. C.; Lin, C. I.; Pelton, J. G.; Chang, M. C. Y. Nature 2019, 567, 420.

(33). Bunno, R.; Awakawa, T.; Mori, T.; Abe, I. Angew. Chem. Int. Ed. 2021, 60, 15827.

(34) Zakharian, T. Y.; Di Costanzo, L.; Christianson, D. W. J. Am. Chem. Soc. 2008, 130, 17254. 